\title{
Transfer of an unknown quantum state, quantum networks, and memory
}

\author{
Asoka Biswas and G. S. Agarwal \\ Physical Research Laboratory, Navrangpura, Ahmedabad 380 009, India \\ (Received 13 May 2003; revised manuscript received 19 May 2004; published 27 August 2004)
}

\begin{abstract}
We present a protocol for transfer of an unknown quantum state. The protocol is based on a two-mode cavity interacting dispersively in a sequential manner with three-level atoms in the $\Lambda$ configuration. We propose a scheme for quantum networking using an atomic channel. We investigate the effect of cavity decoherence in the entire process. Further, we demonstrate the possibility of an efficient quantum memory for arbitrary superposition of two modes of a cavity containing one photon.
\end{abstract}

DOI: 10.1103/PhysRevA.70.022323

PACS number(s): 03.67.Hk, 42.50.Pq

\section{INTRODUCTION}

In the quantum information theory [1], transfer of information in the form of a coherently prepared quantum state is essential. One can transfer a quantum state either by the method of teleportation [2] or through quantum networking. The basic idea behind a quantum network is to transfer a quantum state from one node to another node with the help of a career (a quantum channel) such that it arrives intact. In between, one has to perform a process of quantum state transfer (QST) to transfer the state from one node to the career and again from the career to the destination node. There have been some proposals [3] for quantum networking using cavity-QED, where two atoms trapped inside two spatially separated cavities serve the purpose of two nodes. In Ref. [3], the task was to transfer the state of one atom into the other via the process of QST between the atom and photon, where the latter is used as a career. The photon carries the information through either free space or an optical fiber between the cavities, and the success depends on the probabilistic detection of photons or adiabatic passage through the cavities. We note that, though it may be difficult to beat the communication with photons, it is always interesting to explore the alternatives. In fact, very recently, quantum network using a linear $X Y$ chain of $N$ interacting qubits was proposed. In this proposal, the quantum state can be transferred from the first qubit to the $N$ th qubit within microscopic distance by preengineering interqubit interactions [4].

Further, storage of quantum states is also an important issue. There have been several proposals for quantum memory. For example, recent proposals [5,6] have shown how to transfer the field state into atomic coherence by the adiabatic technique and again retrieve the same through the method of adiabatic following [5] or using the teleportation technique [6]. Quantum memory of the individual polarization state into a collective atomic ensemble has been proposed [7]. Initially, an entangled state of two pairs of atomic ensembles is prepared, where the single-photon polarization state is stored through a process similar to teleportation. Though the information can be transferred back to the photon state, the protocol only succeeds with a probability $1 / 4$. Decoherence-free memory of one qubit in a pair of trapped ions has also been experimentally demonstrated [8]. Maître et al. [9] have proposed a quantum memory, where the quantum information on the superposition state of a two-level atom was stored in a cavity as a superposition of zero- and one-photon Fock states. The holding time of such memories is generally limited by the cavity decay time.

In this paper, we propose a scheme for QST to transfer the unknown state of one atom to another atom where the atoms are not directly interacting with each other. Note that by direct spin interaction of the $\vec{S}_{1} \cdot \vec{S}_{2}$ kind, the quantum state could be transferred from one atom to another within a microscopic range. In the present scheme, we show how a similar kind of interaction between two atoms can be mediated via a cavity. Thus the atomic state can be transferred from one atom to another in the mesoscopic range.

We extend our idea of QST to a quantum network, where we transfer the state of one cavity to another spatially separated cavity. For this we use long-lived atoms as career, and make use of the QST process to transfer the state of the cavity to an atom and again to the target cavity. Our protocol for quantum networking provides a deterministic way to transfer the quantum state between the cavities. This protocol does not require any kind of probability arguments based on the outcome of a measurement. Further, we propose the realization of a quantum memory of arbitrary superposition of two modes of a cavity which contains only one photon. This superposition state can be stored in the long-lived states of the neutral atoms and retrieved in another two-mode cavity later, deterministically. Our proposal relies on the technological advances and realizations as described in Ref. [10].

The structure of the paper is as follows. In Sec. II, we describe the model and provide the relevant equations. In Sec. III, we discuss how transfer of an unknown quantum state can be performed between two atoms. We provide an estimate of possible decoherence in this process due to cavity decay. In Sec. IV, we extend our scheme to quantum networks and quantum memory.

\section{MODEL CONFIGURATION}

To describe how the QST protocol works, we consider a three-level atom in the $\Lambda$ configuration interacting with a two-mode cavity (see Fig. 1). The modes with annihilation operators $a$ and $b$ interact with the $|e\rangle \leftrightarrow|g\rangle$ and $|e\rangle \leftrightarrow|f\rangle$ transitions, respectively. The Hamiltonian under the rotating wave approximation can be written as 


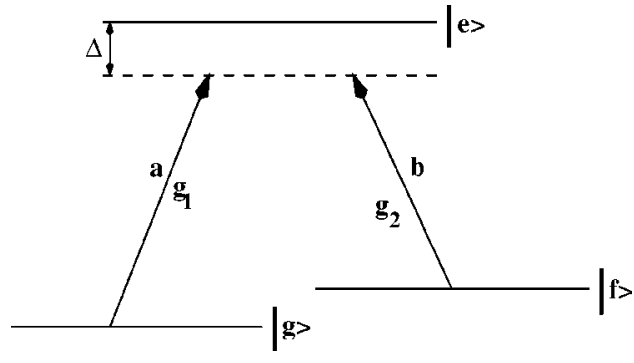

FIG. 1. Three-level atomic configuration with levels $|g\rangle,|e\rangle$, and $|f\rangle$ interacting with two orthogonal modes of the cavity, described by operators $a$ and $b$. Here $g_{1}$ and $g_{2}$ represent the atom-cavity coupling of the $a$ and $b$ modes with the corresponding transitions and $\Delta$ is the common one-photon detuning.

$$
\begin{aligned}
H= & \hbar\left[\omega_{e g}|e\rangle\left\langle e\left|+\omega_{f g}\right| f\right\rangle\langle f|+\omega_{1} a^{\dagger} a+\omega_{2} b^{\dagger} b+\left\{g_{1}|e\rangle\langle g| a\right.\right. \\
& \left.\left.+g_{2}|e\rangle\langle f| b+\text { H.c. }\right\}\right],
\end{aligned}
$$

where $\omega_{l g}(l \in e, f)$ is the atomic transition frequency, $\omega_{i}(i$ $\in 1,2)$ is the frequency of the cavity modes $a$ and $b$, and $g_{i}$ is the atom-cavity coupling constant. We assume $g_{i}$ to be real.

We work under the two-photon resonance condition and assume large single-photon detuning. After adiabatically eliminating the excited level $|e\rangle$ in the large detuning domain, we derive an effective Hamiltonian describing the system of Fig. 1,

$$
\begin{aligned}
H_{\mathrm{eff}}= & -\frac{\hbar g^{2}}{\Delta}\left[|g\rangle\left\langle g\left|a^{\dagger} a+\right| f\right\rangle\langle f| b^{\dagger} b\right] \\
& -\frac{\hbar g^{2}}{\Delta}\left[|g\rangle\left\langle f\left|a^{\dagger} b+\right| f\right\rangle\langle g| a b^{\dagger}\right],
\end{aligned}
$$

where $\Delta=\omega_{e g, f}-\omega_{1,2}$ is the common one-photon detuning of the cavity modes and $g_{1}=g_{2}=g(\ll \Delta)$. The condition $g_{1}=g_{2}$ can be satisfied by proper choice as we can choose appropriate transitions in atomic systems, frequencies, etc. Note that if one considers the levels $|g\rangle$ and $|f\rangle$ as Zeeman sublevels, then these conditions are automatically satisfied. In that case, we may consider the two modes of the cavity as two orthogonal polarization states of a photon. Now note that the first two terms in Eq. (2) represent the self-energy terms and the last two terms give the interaction leading to a transition from the initial state to the final state. The probability amplitudes of relevant basis states $|g\rangle|n, \mu\rangle$ and $|f\rangle|n-1, \mu+1\rangle$ in the state vector

$$
|\psi(t)\rangle=d_{g}(t)|g, n, \mu\rangle+d_{f}(t)|f, n-1, \mu+1\rangle
$$

are given by

$$
\begin{aligned}
& d_{g}(t)=\frac{\sqrt{n} X Y}{n+\mu+1}+d_{g}(0), \\
& d_{f}(t)=\frac{\sqrt{\mu+1} X Y}{n+\mu+1}+d_{f}(0),
\end{aligned}
$$

where $X=\sqrt{n} d_{g}(0)+\sqrt{\mu+1} d_{f}(0), \quad Y=\exp \left[i g^{2}(n+\mu+1) t / \Delta\right]$ -1 , and $n$ and $\mu$ are the respective photon numbers in the modes $a$ and $b$. We note that the effective interaction (2) can be seen as an interaction between two qubits defined via the atomic variables and field variables

$$
\begin{gathered}
S^{+}=|f\rangle\left\langle g\left|, \quad S^{-}=\right| g\right\rangle\langle f|, \quad S^{z}=\frac{1}{2}(|f\rangle\langle f|-| g\rangle\langle g|) ; \\
R^{+}=a^{\dagger} b, \quad R^{-}=a b^{\dagger}, \quad R^{z}=\frac{1}{2}\left(a^{\dagger} a-b^{\dagger} b\right) .
\end{gathered}
$$

In the single-photon space, the field operators $R^{ \pm}, R^{z}$ satisfy spin-1/2 algebra and thus the interaction (2) can be written as an interaction between two qubits,

$$
H_{\mathrm{eff}} \equiv-\frac{\hbar g^{2}}{\Delta}\left(R^{+} S^{-}+R^{-} S^{+}-2 R^{z} S^{z}\right)
$$

In view of the above form of the effective interaction, we conclude that our system of Fig. 1 can be used for a number of quantum logic operations.

\section{QUANTUM STATE TRANSFER PROTOCOL}

We next demonstrate how the dynamics of an atom in a two-mode cavity can be used to implement the QST protocol. Hereafter, we will use the term " $\pi$ pulse" to denote an equivalent traversal time $T$ of the atom through the cavity such that $2 g^{2} T / \Delta=\pi$. The time $T$ could be controlled by selecting the atomic velocity.

We assume that the atom $A$ is initially in an unknown state,

$$
|i\rangle_{A}=\alpha|g\rangle_{A}+\beta|f\rangle_{A},
$$

where $\alpha$ and $\beta$ are unknown arbitrary coefficients. The state $|i\rangle_{A}$ of atom $A$ is to be transferred to another atom $B$ which is elsewhere. Preparing the cavity in a state $|0,1\rangle$ (i.e., initially one photon in the $b$ mode), we send the atom $A$ through the cavity for a certain time which is equivalent to a $\pi$ pulse. After atom $A$ comes out of the cavity, atom $B$ in state

$$
\left|i^{\prime}\right\rangle=\alpha^{\prime}|g\rangle+\beta^{\prime}|f\rangle
$$

is sent through the cavity. Here $\alpha^{\prime}$ and $\beta^{\prime}$ are arbitrary coefficients and need not be known. Atom $B$ also experiences a $\pi$ pulse during the interaction with the cavity. The entire process can be described as follows:

$$
\begin{aligned}
|i\rangle_{A} & |0,1\rangle \\
\downarrow & \pi \text { pulse on atom } A \\
|g\rangle_{A} & (\alpha|0,1\rangle-\beta|1,0\rangle) \\
\downarrow & B \text { atom enters } \\
|g\rangle_{A} & \left|i^{\prime}\right\rangle_{B}(\alpha|0,1\rangle-\beta|1,0\rangle) \\
\downarrow & \pi \text { pulse on atom } B \\
|g\rangle_{A} & |i\rangle_{B}\left(\alpha^{\prime}|0,1\rangle-\beta^{\prime}|1,0\rangle\right) .
\end{aligned}
$$




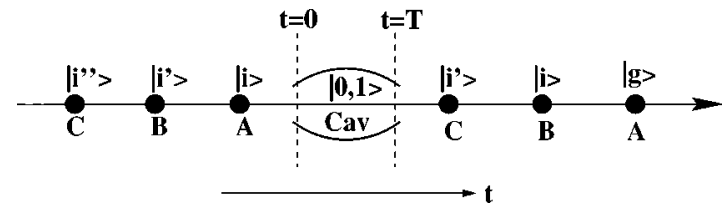

FIG. 2. Schematic diagram for the QST protocol for a number of atoms interacting with the two-mode cavity in a sequential manner for a time $T=\Delta \pi / 2 g^{2}$.

If one prepares the cavity initially in state $|1,0\rangle$, then following a similar sequence to the above, the final state will be $-|f\rangle_{A}|i\rangle_{B}\left(\alpha^{\prime}|0,1\rangle-\beta^{\prime}|1,0\rangle\right)$. Note that atom $B$ has already acquired the state $|i\rangle$ of atom $A$, i.e., the state $|i\rangle$ is transferred from atom $A$ to atom $B$.

More generally, our QST protocol can be written as

$$
|i\rangle_{A}\left|i^{\prime}\right\rangle_{B}(\gamma|0,1\rangle+\delta|1,0\rangle)_{\mathrm{cav}} \stackrel{U(\pi)}{\rightarrow}(\gamma|g\rangle-\delta|f\rangle)_{A}|i\rangle_{B}|\psi\rangle_{\mathrm{cav}},
$$

where $\quad U(\pi)=U_{A}(\pi) U_{B}(\pi), \quad U_{k}(\pi) \quad(k \in A, B) \quad[=\exp$ $\left.\left\{-i H_{\text {eff }} T / \hbar\right\}\right]$ denotes the $\pi$-pulse operation on the atom $k$, and

$$
|\psi\rangle_{\mathrm{cav}}=\alpha^{\prime}|0,1\rangle_{\mathrm{cav}}-\beta^{\prime}|1,0\rangle_{\mathrm{cav}} .
$$

Our protocol has interesting features: (a) the initial states of the atoms can be arbitrary, and (b) the field state can also be an arbitrary superposition of $|0,1\rangle$ and $|1,0\rangle$. Note that in the case of a two-level atom interacting with a resonant singlemode cavity, the QST protocol from one atom to another atom has difficulties associated with a relative phase which can be changed either by using a conditional phase shift which is essentially a two-qubit operation [see Eq. (3.8) of Ref. [10]] or by applying a resonant microwave field to the atomic qubit.

We note that if the initial state of the atom $B$ is $|g\rangle$ (or $|f\rangle$ ) and the cavity is initially in state $|0,1\rangle$ (or $|1,0\rangle$ ), then we can not only transfer the state of atom $A$ to $B$, but we also can interchange the states between them. However, the QST protocol described here cannot be interpreted as a SWAP gate. As in the usual version of a quantum gate, atoms $A$ and $B$ must interact with the field simultaneously. We also note that, in the process of coherence transfer between two atoms using, for example, the scheme of Ref. [11], the atoms must be addressed by the pulses simultaneously, which is basically a local interaction. In the present protocol, the atoms interact with the $\pi$ pulse in a sequential manner. This is essentially a nonlocal process.

Extending the idea of QST described above to a number of atoms, we can transfer the state of any atom to the consecutive atom. This means that if we consider a sequel of atoms, then the state of any atom can be transferred to the consecutive atom which will pass the cavity after the former leaves the cavity. The procedure of transfer of atomic states to consecutive atoms has been shown schematically in Fig. 2 . Here the atoms $A, B, C$, etc. are sent through another identical bimodal cavity in initial state $|0,1\rangle$. After passing through this cavity, atom $C$ is again prepared in state $|i\rangle$. Thus, using a second cavity in this way, we can transfer the state of the first atom $A$ to a third atom $C$. Clearly, if we used $n$ number of cavities in this sequence, we could transfer the state of atom $A$ to the $(n+1)$ th atom in the sequence.

\section{Effects of decoherence: Fidelity of the QST protocol}

Decoherence is a strong limiting factor in the realization of any quantum computational protocol. The interaction of the atom and the cavity with the environment causes them to decay and results in decoherence. Thus, one has to consider the effect of decoherence to examine with how much efficiency the desired outcome can be produced. These calculations can be done in the density-matrix framework using the following Liouville equation:

$$
\begin{aligned}
\dot{\rho}= & -\frac{i}{\hbar}\left[H_{\mathrm{eff}}, \rho\right]-\kappa_{a}\left(a^{\dagger} a \rho-2 a \rho a^{\dagger}+\rho a^{\dagger} a\right) \\
& -\kappa_{b}\left(b^{\dagger} b \rho-2 b \rho b^{\dagger}+\rho b^{\dagger} b\right),
\end{aligned}
$$

where $\kappa_{a}$ and $\kappa_{b}$ are the decay constants of the two modes and $H_{\text {eff }}$ is given by Eq. (2).

In the present case, to investigate the effect of decoherence, let us consider a possible scheme. We consider $|g\rangle$ and $|f\rangle$ to be the Rydberg levels as in Haroche's experiments. In that case, we can use a bimodal microwave cavity like the one used by Haroche's group. We use parameters similar to those in the experiments by Haroche and his co-workers. If the cavity coupling constant $g$ is $2 \pi \times 50 \mathrm{kHz}$ and the cavity decay constant $\kappa_{a}=\kappa_{b}=\kappa$ for each mode is $2 \pi \times 100 \mathrm{~Hz}$, then $\kappa / g=0.002$. Further, for $\Delta=10 g$, we calculate the cavity interaction time to be $50 \mu \mathrm{s}$ for a $\pi$ pulse, which is consistent with the interaction time possible to achieve in a microwave experiment. One sends the atoms with a velocity $\sim 10^{2} \mathrm{~cm} \mathrm{~s}^{-1}$ through a few-cm-long cavity to achieve this interaction time. Using these parameters, we calculate the fidelity $F$ that the first step of the evolution (9) occurs. The variation of $F(T)$ with the decay constant $\kappa$ is shown in Fig. 3(a), where $T$ is the interaction time of the atom with the cavity. Note that the probability that the state of atom $A$ is transferred to the cavity remains more than $90 \%$ for $\kappa$ $=0.002 \mathrm{~g}$. We next show [see Fig. 3(b)] the variation of the fidelity $F(2 T+\tau)$ of the entire process (9) to occur with the time delay $\tau$ between the atoms $A$ and $B$ for $\kappa=0.002 g$. It is clear that the probability that the atom $B$ acquires the desired state remains above $80 \%$ even at $g \tau=20(\equiv \tau \approx 63 \mu$ s).

\section{EXTENSIONS OF QUANTUM STATE TRANSFER PROTOCOL}

\section{A. Quantum networks}

Now we show how the above QST protocol can be made useful in preparing a quantum network, in which long-lived atomic states are used to communicate between the two nodes of the network. We assume that there are two identical two-mode cavities $C_{1}$ and $C_{2}$, which are considered as two nodes of the network. Let us consider that the cavity $C_{1}$ is initially in a state $|0,1\rangle$. To prepare this cavity in a superposition state, 

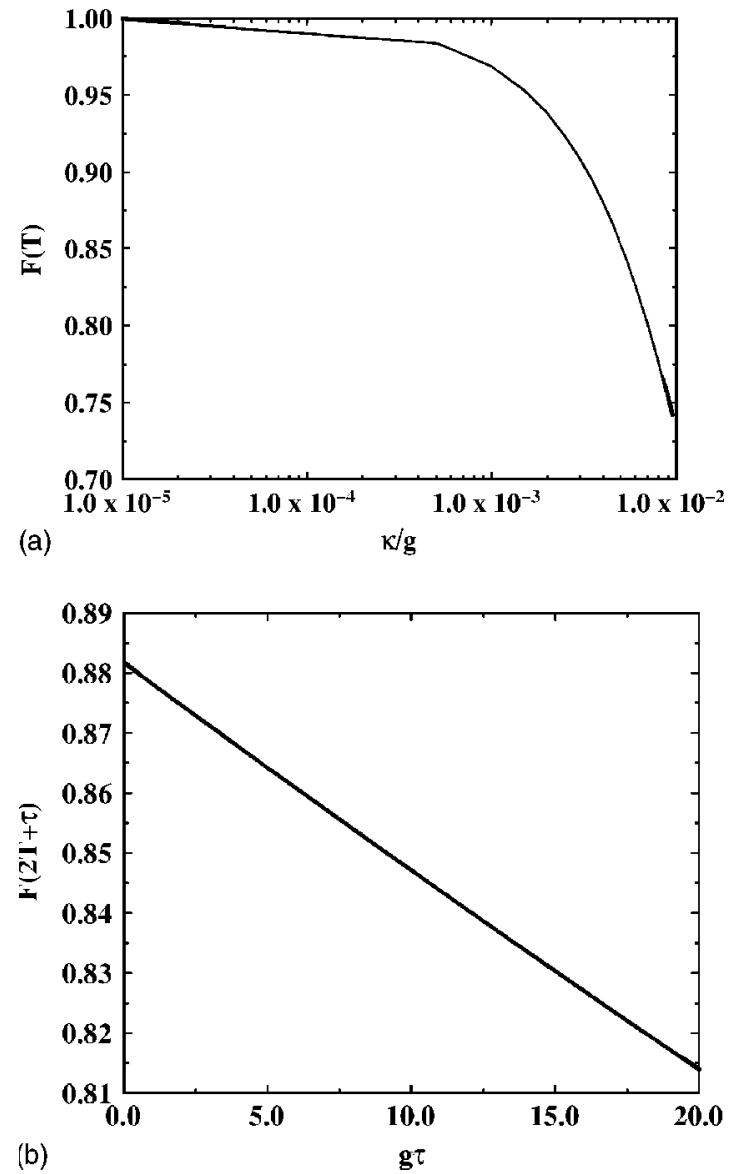

FIG. 3. (a) Variation of the fidelity $F(T)$ of mapping the state of the atom $A$ in the cavity $C_{1}$ with $\kappa / g$. We have assumed that the cavity decay rates are the same for both the modes and $\Delta=10 \mathrm{~g}$. (b) Variation of the fidelity $F$ calculated at time $2 T+\tau$, with the time delay $\tau$ between the atoms for $\kappa=0.002 g$ and $\Delta=10 g$.

$$
|E\rangle_{\mathrm{cav}}=\alpha|0,1\rangle_{\mathrm{cav}}-\beta|1,0\rangle_{\mathrm{cav}},
$$

we send an atom $A$ in state $|i\rangle$ through the cavity (see Fig. 4) such that the atom $A$ experiences a $\pi$ pulse. Now our goal is to transfer this cavity state $|E\rangle_{\text {cav }}$ to the other node $C_{2}$. For that we send a second atom $B$ through the cavity $C_{1}$ after $A$ comes out of it. We see that the atom $B$ is prepared in state $|i\rangle$ through the evolution (9). This atom is now sent through the second node $C_{2}$ which is initially in state $|0,1\rangle$. In this way, the state $|E\rangle_{\text {cav }}$ of node $C_{1}$ is transferred to the node $C_{2}$.

Extending the above idea to a number of distant nodes (cavities), we thus can transfer the state $|E\rangle_{\text {cav }}$ from one node to another node of the proposed quantum network via a quantum channel (atom). For example, to send this state $|E\rangle_{\text {cav }}$ from $C_{2}$ to another node (say, $C_{3}$ ), we can send a third atom $C$ through these two nodes subsequently.

We emphasize that our protocol of quantum networking is distinct from the teleportation protocol of Davidovich et al. [12]. Their protocol depends on the Bell state measurements, whereas in our protocol no Bell measurement is ever made.

We further note that the present scheme can be used to spread entanglement between two distant cavities. For this, one first sends an atom $A$ in state $|g\rangle$ through the first cavity

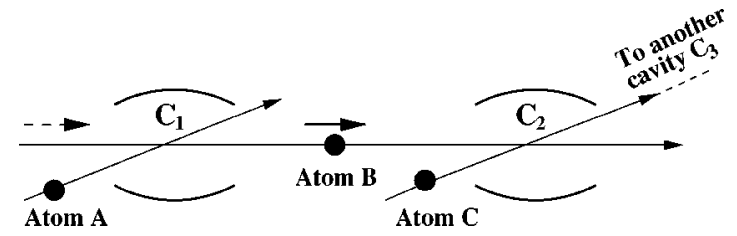

FIG. 4. Schematic diagram for the quantum network between distant cavities via the atomic channel. Description of the figure is in the text.

$C_{1}$ prepared initially in the state $|1,0\rangle$ such that the atom experiences a $\pi / 2$ pulse $\left(2 g^{2} T / \Delta=\pi / 2\right)$. This would prepare the atom and the cavity in the following entangled state:

$$
|\Psi\rangle_{A C_{1}}=\frac{1}{\sqrt{2}} e^{i \pi / 2}\left(|g\rangle_{A}|1,0\rangle_{1}+|f\rangle_{A}|0,1\rangle_{1}\right) .
$$

Next the atom passes through a second cavity $C_{2}$ initially in the state $|0,1\rangle$ and experiences a $\pi$ pulse. Thus, at the end of this process, the two cavities are prepared in an entangled state of two modes as

$$
|\Psi\rangle_{C_{1} C_{2}}=\frac{1}{\sqrt{2}} e^{i \pi / 2}\left[|1,0\rangle_{1}|0,1\rangle_{2}-|0,1\rangle_{1}|1,0\rangle_{2}\right] .
$$

Clearly one can spread entanglement between the atom and the cavity to another distant cavity. Note that in our proposal, entanglement is created between the modes of the two different cavities. The entanglement between two modes of a single cavity has been produced in [13].

\section{B. Storage and retrieval of an arbitrary superposition state of two modes of a cavity}

We now discuss how the present $\pi$-pulse technique can be used to prepare an efficient quantum memory for arbitrary superposition of two cavity modes, where there is only one photon present in either mode. Let us consider a two-mode cavity which is in a superposition state of two modes [see Eq. (13)],

$$
|E\rangle_{\mathrm{cav}}=\alpha|0,1\rangle_{\mathrm{cav}}-\beta|1,0\rangle_{\mathrm{cav}},
$$

where $\alpha$ and $\beta$ are known coefficients. Now we send an atom in state (8) through the cavity. Applying a $\pi$ pulse on it, we can map the superposition of $|E\rangle_{\text {cav }}$ into the state of the atom. This procedure can be written as

$$
\left|i^{\prime}\right\rangle|E\rangle_{\mathrm{cav}} \rightarrow-|i\rangle|\psi\rangle_{\mathrm{cav}},
$$

where $|i\rangle=\alpha|g\rangle+\beta|f\rangle$ and $|\psi\rangle_{\text {cav }}$ is given by Eq. (11). Because, the states $|g\rangle$ and $|f\rangle$ of the atom are radiatively longlived, information about the state of the cavity can be stored inside the atom for a sufficiently long time. To retrieve this information into the cavity, we prepare a second cavity in either of the states $|0,1\rangle$ or $|1,0\rangle$ and send the atom in state $|i\rangle$ through the cavity. Upon applying a $\pi$ pulse, the cavity can again be prepared in the superposition state as before. The retrieval of superposition can be shown as 


$$
|i\rangle|0,1\rangle_{\mathrm{cav}} \rightarrow|g\rangle|E\rangle_{\mathrm{cav}}, \quad|i\rangle|1,0\rangle_{\mathrm{cav}} \rightarrow-|f\rangle|E\rangle_{\mathrm{cav}} .
$$

We should mention here that the quantum memory proposed here for the cavity state is expected to work better since the information is being stored inside the long-lived atomic states $|g\rangle$ and $|f\rangle$. However, the transfer time of the cavity state to the atom is limited by the cavity holding time and the atom must stop interacting with the cavity before it decays. We also note that if the two modes are degenerate and correspond to two states of circular polarizations, then Eq. (16) can be viewed as a superposition of two polarization states of a photon. In such a case, our proposal corresponds to storage and retrieval of the polarization states of a photon.

\section{CONCLUSION}

In conclusion, we have presented a protocol for the transfer of a quantum state from one atom to another atom. This protocol can be extended to a number of atoms passing through sequential cavities and thus one can set up a quantum network. We have further shown how an efficient quantum memory of arbitrary superposition of two cavity modes can be built up. Our proposals have certain advantages as we work with long-lived states of atoms. We provide a proper estimate of the efficiency of the state transfer protocol against cavity decoherence.
[1] M. Keyl, Phys. Rep. 369, 431 (2002); A. Galindo and M. A. Martín-Delgado, Rev. Mod. Phys. 74, 347 (2002).

[2] C. H. Bennett et al., Phys. Rev. Lett. 70, 1895 (1993).

[3] J. I. Cirac et al., Phys. Rev. Lett. 78, 3221 (1997); S. J. van Enk, J. I. Cirac, and P. Zoller, ibid. 78, 4293 (1997); T. Pellizzari, ibid. 79, 5242 (1997).

[4] M. Christandl, N. Datta, A. Ekert, and A. J. Landahl, Phys. Rev. Lett. 92, 187902 (2004).

[5] D. F. Phillips et al., Phys. Rev. Lett. 86, 783 (2001); M. Fleischhauer and M. D. Lukin, Phys. Rev. A 65, 022314 (2002).

[6] A. E. Kozhekin, K. Mølmer, and E. Polzik, Phys. Rev. A 62, 033809 (2000); C. Schori, B. Julsgaard, J. L. Sørensen, and E.
S. Polzik, Phys. Rev. Lett. 89, 057903 (2002).

[7] G.-P. Guo and G.-C. Guo, Phys. Lett. A 318, 337 (2003).

[8] D. Kielpinski et al., Science 291, 1013 (2001).

[9] X. Maître et al., Phys. Rev. Lett. 79, 769 (1997).

[10] J. M. Raimond, M. Brune, and S. Haroche, Rev. Mod. Phys. 73, 565 (2001).

[11] T. Pellizzari, S. A. Gardiner, J. I. Cirac, and P. Zoller, Phys. Rev. Lett. 75, 3788 (1995).

[12] L. Davidovich, N. Zagury, M. Brune, J. M. Raimond, and S. Haroche, Phys. Rev. A 50, R895 (1994).

[13] A. Rauschenbeutel et al., Phys. Rev. A 64, 050301 (2001). 